\title{
Persistent memory of diffusing particles
}

\author{
N. Suciu, ${ }^{1,2, *}$ C. Vamoş, ${ }^{2}$ F. A. Radu, ${ }^{3,4}$ H. Vereecken, ${ }^{5}$ and P. Knabner ${ }^{1}$ \\ ${ }^{1}$ Chair for Applied Mathematics I, Friedrich-Alexander University Erlangen-Nuremberg, Erlangen, Germany \\ ${ }^{2}$ Tiberiu Popoviciu Institute of Numerical Analysis, Romanian Academy, Cluj Napoca, Romania \\ ${ }^{3}$ Computational Hydrosystems, Helmholtz. Center for Environmental Research-UFZ, Leipzig, Germany \\ ${ }^{4}$ Institute of Geosciences, University of Jena, Jena, Germany \\ ${ }^{5}$ Research Center Jülich, Agrosphere Institute ICG-IV, Jülich, Germany
}

(Received 30 September 2009; published 28 December 2009)

\begin{abstract}
The variance of the advection-diffusion processes with variable coefficients is exactly decomposed as a sum of dispersion terms and memory terms consisting of correlations between velocity and initial positions. For random initial conditions, the memory terms quantify the departure of the preasymptotic variance from the time-linear diffusive behavior. For deterministic initial conditions, the memory terms account for the memory of the initial positions of the diffusing particles. Numerical simulations based on a global random walk algorithm show that the influence of the initial distribution of the cloud of particles is felt over hundreds of dimensionless times. In case of diffusion in random velocity fields with finite correlation range the particles forget the initial positions in the long-time limit and the variance is self-averaging, with clear tendency toward normal diffusion.
\end{abstract}

DOI: 10.1103/PhysRevE.80.061134

PACS number(s): 05.60.Cd, 02.50.Ey, 05.10.Gg, 05.10.Ln

\section{INTRODUCTION}

A stochastic process has diffusive behavior when its variance is a linear-time function. The simplest example is the advection-diffusion process with constant coefficients described by a Gaussian normalized concentration (one-time probability density) $c(x, t)=(4 \pi D t)^{-1 / 2} \exp \left[-(x-V t)^{2} / 4 D t\right]$. The mean $\mu(t)$ and the variance $s(t)$ are both linear-time functions,

$$
\begin{gathered}
\mu(t)=\int x c(x, t) d x=V t, \\
s(t)=\int[x-\mu(t)]^{2} c(x, t) d x=2 D t,
\end{gathered}
$$

and their time derivatives give the constant velocity and diffusion coefficients of the process [1],

$$
V=\frac{d}{d t} \mu(t), \quad D=\frac{d}{2 d t} s(t) .
$$

In this case, the diffusion coefficient $D$ describes both the shape of the Gaussian distribution $c(x, t)$ and the width of the diffusion front $s(t)$. For transport in systems with space-timevariable properties, the mean and the variance are no longer related with the variable velocity and diffusion coefficients by the simple relations [Eq. (1)].

In Sec. II we derive general relations between coefficients and the covariance of the advection-diffusion process. These relations show that the variable diffusion coefficients contribute to the covariance of the process by the time integral of their expected values. The variability of the velocity instead yields two different contributions: dispersion terms, expressed by Taylor-Kubo relations as time integrals of the

\footnotetext{
*suciu@am.uni-erlangen.de
}

Lagrangian velocity correlation, and memory terms, accounting for correlations between initial positions of the diffusing particles and their Lagrangian velocity. In Sec. III we prove that the extinction of the memory terms in the long-time limit is a necessary condition for the occurrence of the normal diffusion.

Statistical physics approaches for spatially homogeneous systems $[2,3]$ or for transport in inhomogeneous and rapidly fluctuating velocity fields, as in plasma physics [4], are concerned with memory effects which characterize the departure of the process from diffusive behavior. Such effects are related to the occurrence of the anomalous transport $[4,5]$ and are usually described by nonlocal equations, containing memory kernels which govern the velocity autocorrelation function or the ensemble average of a transported scalar [6-9]. Our approach to investigate memory effects, though related to those cited above, is somehow simpler and straightforward. Instead of describing memory effects by convolution memory kernels $[2,7,8]$, we investigate the time behavior of the memory terms. The latter can be expressed in general for either continuous time-space or discrete transport processes as correlations between displacements of the diffusing particles and their initial positions. Such correlations relate the linear-time diffusive behavior of the variance to the lose of memory of the past itinerary of the particles. This condition of diffusive behavior generalizes to the case of variable coefficients the independence of increments of the Wiener process (Sec. III).

Our approach allows one to treat in a unitary way memory effects manifested by departure from normal diffusive behavior and the memory of the initial positions of the particles. In $\mathrm{Sec}$ IV we show, via a numerical experiment, that memory terms also quantify the persistent influence of the deterministic initial conditions on the variance of the transport process and its departure from model statistical descriptions by ensemble averages. In particular, it is shown that the selfaveraging behavior of the effective coefficients of diffusion in random velocity fields $[10,11]$ is clearly related to the 
destruction of the memory terms. Section V summarizes the results and outlines directions for further work.

\section{DISPERSION AND MEMORY TERMS}

We consider, for the beginning, the continuous diffusion process with space-time-variable diffusion coefficients $D_{i j}(\mathbf{x}, t)$ and velocity components $V_{i}(\mathbf{x}, t), i, j=1,2,3$. The density of the transition probability $g\left(\mathbf{x}, t \mid \mathbf{x}_{0}, t_{0}\right)$ is the solution of the Fokker-Planck equation

$$
\partial_{t} g+\partial_{x_{i}}\left(V_{i} g\right)=\partial_{x_{i}} \partial_{x_{j}}\left(D_{i j} g\right)
$$

for the initial condition $g\left(\mathbf{x}, t_{0} \mid \mathbf{x}_{0}, t_{0}\right)=\delta\left(\mathbf{x}-\mathbf{x}_{0}\right)$. The time evolution of the normalized concentration is given by

$$
c(\mathbf{x}, t)=\int g\left(\mathbf{x}, t \mid \mathbf{x}_{0}, t_{0}\right) c\left(\mathbf{x}_{0}, t_{0}\right) d \mathbf{x}_{0},
$$

where $c\left(\mathbf{x}_{0}, t_{0}\right)$ is the initial concentration and the integral extends over the entire space.

A diffusion process is defined by the following conditions uniformly satisfied in $\mathbf{x}$ and $t$ for all $\epsilon>0[1,12,13]$ :

$$
\begin{gathered}
\lim _{\Delta t \rightarrow 0} \frac{1}{\Delta t} \int_{\left|\mathbf{x}^{\prime}-\mathbf{x}\right| \geq \epsilon} g\left(\mathbf{x}^{\prime}, t+\Delta t \mid \mathbf{x}, t\right) d \mathbf{x}^{\prime}=0 \\
V_{i}(\mathbf{x}, t)=\lim _{\Delta t \rightarrow 0} \frac{1}{\Delta t} \times \int_{\left|\mathbf{x}^{\prime}-\mathbf{x}\right|<\epsilon}\left(x_{i}^{\prime}-x_{i}\right) g\left(\mathbf{x}^{\prime}, t+\Delta t \mid \mathbf{x}, t\right) d \mathbf{x}^{\prime}, \\
D_{i j}(\mathbf{x}, t)=\frac{1}{2} \lim _{\Delta t \rightarrow 0} \frac{1}{\Delta t} \times \int_{\left|\mathbf{x}^{\prime}-\mathbf{x}\right|<\epsilon}\left(x_{i}^{\prime}-x_{i}\right)\left(x_{j}^{\prime}-x_{j}\right) \\
\quad \times g\left(\mathbf{x}^{\prime}, t+\Delta t \mid \mathbf{x}, t\right) d \mathbf{x}^{\prime} .
\end{gathered}
$$

Condition (4) ensures the continuity with probability 1 for the trajectories of the diffusion process. The velocity components $V_{i}$ and the diffusion coefficients $D_{i j}$ of the FokkerPlanck equation [Eq. (2)] are defined in Eqs. (5) and (6) by means of the first two local moments (computed on spheres of radius $\epsilon$ ) of the transition probability of the process starting at $(\mathbf{x}, t)$. Since the mean and the variance in Eq. (1) are integrals over the entire space, it follows that for processes with constant coefficients two more conditions are fulfilled for every $\epsilon>0$,

$$
\begin{aligned}
& \lim _{\Delta t \rightarrow 0} \frac{1}{\Delta t} \int_{\left|\mathbf{x}^{\prime}-\mathbf{x}\right| \geq \epsilon} x_{i}^{\prime} g\left(\mathbf{x}^{\prime}, t+\Delta t \mid \mathbf{x}, t\right) d \mathbf{x}^{\prime}=0, \\
& \lim _{\Delta t \rightarrow 0} \frac{1}{\Delta t} \int_{\left|\mathbf{x}^{\prime}-\mathbf{x}\right| \geq \epsilon} x_{i}^{\prime} x_{j}^{\prime} g\left(\mathbf{x}^{\prime}, t+\Delta t \mid \mathbf{x}, t\right) d \mathbf{x}^{\prime}=0 .
\end{aligned}
$$

Conditions (7) and (8) correspond to the physically reasonable assumption that the first two moments are finite at finite times [12]. In the following we restrict the solutions of the Fokker-Planck equation [Eq. (2)] to the class of transition probabilities obeying Eqs. (7) and (8). In this case, the integrals in the definitions [Eqs. (5) and (6)] of the coefficients can be extended over the entire space [12].
Using the definitions [Eqs. (5) and (6)] of the coefficients and the constraints on the transition probability [Eqs. (4), (7), and (8)], we computed in Appendix A the components of the mean $\mu_{i}$ and of the covariance $s_{i j}$, which have the following explicit dependence on the coefficients of the Fokker-Planck equation [Eq. (2)]:

$$
\mu_{i}\left(t, t_{0}\right)=\int x_{i} c(\mathbf{x}, t) d \mathbf{x}=\mu_{i}\left(t_{0}\right)+\int_{t_{0}}^{t} \bar{V}_{i}\left(t^{\prime}\right) d t^{\prime},
$$

$$
\begin{aligned}
s_{i j}\left(t, t_{0}\right)= & \int\left[x_{i}-\mu_{i}(t)\right]\left[x_{j}-\mu_{j}(t)\right] c(\mathbf{x}, t) d \mathbf{x} \\
= & s_{i j}\left(t_{0}\right)+2 \int_{t_{0}}^{t} d t^{\prime} \int D_{i j}\left(\mathbf{x}, t^{\prime}\right) c\left(\mathbf{x}, t^{\prime}\right) d \mathbf{x}+s_{u, i j}\left(t, t_{0}\right) \\
& +m_{i j}\left(t, t_{0}\right)
\end{aligned}
$$

$$
\begin{aligned}
s_{u, i j}\left(t, t_{0}\right)= & \int_{t_{0}}^{t} d t^{\prime} \int_{t_{0}}^{t^{\prime}} d t^{\prime \prime} \int c\left(\mathbf{x}_{0}, t_{0}\right) d \mathbf{x}_{0} \\
& \times \iint\left[u_{i}\left(\mathbf{x}^{\prime}, t^{\prime \prime}\right) u_{j}\left(\mathbf{x}, t^{\prime}\right)+u_{j}\left(\mathbf{x}^{\prime}, t^{\prime \prime}\right) u_{i}\left(\mathbf{x}, t^{\prime}\right)\right] \\
& \times g\left(\mathbf{x}, t^{\prime} \mid \mathbf{x}^{\prime}, t^{\prime \prime}\right) g\left(\mathbf{x}^{\prime \prime}, t^{\prime} \mid \mathbf{x}_{0}, t_{0}\right) d \mathbf{x} d \mathbf{x}^{\prime}, \\
m_{i j}\left(t, t_{0}\right)= & \int_{t_{0}}^{t} d t^{\prime} \int c\left(\mathbf{x}_{0}, t_{0}\right) d \mathbf{x}_{0} \int\left\{\left[x_{0 j}-\mu_{j}\left(t_{0}\right)\right] u_{i}\left(\mathbf{x}, t^{\prime}\right)\right. \\
& \left.+\left[x_{0 i}-\mu_{i}\left(t_{0}\right)\right] u_{j}\left(\mathbf{x}, t^{\prime}\right)\right\} g\left(\mathbf{x}, t^{\prime} \mid \mathbf{x}_{0}, t_{0}\right) d \mathbf{x},
\end{aligned}
$$

where

$$
\bar{V}_{i}(t)=\int V_{i}(\mathbf{x}, t) c(\mathbf{x}, t) d \mathbf{x}
$$

is the mean velocity and $u_{i}(\mathbf{x}, t)=V_{i}(\mathbf{x}, t)-\bar{V}_{i}(t)$ is the velocity fluctuation.

The physical meaning of the contributions [Eqs. (11) and (12)] to the covariance [Eq. (10)] becomes clearer when we express these terms within the Lagrangian framework as averages over the trajectories of the diffusing particles [9]. To this end, we note that for a given diffusion process with sufficiently smooth coefficients [Eqs. (5) and (6)], it is always possible to construct weak solutions of the associated Itô equation with transition probability densities described by the Fokker-Planck equation [Eq. (2)] $[12,13]$. Let us consider a diffusion process $\left\{X_{i}(t), \quad t \geq t_{0}, \quad i=1,2,3\right\}$ with stationary velocity $\mathbf{V}(\mathbf{x})$ and isotropic constant diffusion coefficients $D_{i j}=D$ and the Itô equation

$$
X_{i}(t)=X_{0 i}+\int_{t_{0}}^{t} V_{i}\left[\mathbf{X}\left(t^{\prime}\right)\right] d t^{\prime}+W_{i}\left(t-t_{0}\right),
$$

where $W_{i}\left(t-t_{0}\right)$ is a Brownian motion modeled as a Wiener process with mean zero and variance $2 D\left(t-t_{0}\right)$ [13]. The Itô equation, which when written in differential form $d X_{i}(t)$ $=V_{i}[\mathbf{X}(t)] d t+d W_{i}(t)$ is also referred to as Langevin equation, is often used as model for the movement of diffusing particles in physical systems [1,14-16]. 
In the following we denote averages over trajectories of particles starting at given initial positions and averages with respect to initial distributions of particles by angular brackets with subscripts $D$ and $X_{0}$, respectively, and we pass to a Lagrangian description using the expressions of the probability densities derived in Appendix B. From Eq. (B1) it becomes evident that the mean velocity [Eq. (13)] is the average of the velocity field viewed by particles moving on trajectories, $\bar{V}_{i}(t)=\left\langle V_{i}\left[X_{i}(t)\right]\right\rangle_{D X_{0}}$, i.e., it is the average of the Lagrangian velocity [17]. In the same way, using Eq. (B2), the integrands in Eqs. (11) and (12) are expressed in terms of autocorrelation and correlation with initial positions of the Lagrangian velocity. The diagonal components of the covariance $[\mathrm{Eq} .(10)]$ become

$$
\begin{aligned}
s_{i i}\left(t, t_{0}\right)= & s_{i i}\left(t_{0}\right)+2 D\left(t-t_{0}\right) \\
& +2 \int_{t_{0}}^{t} d t^{\prime} \int_{t_{0}}^{t^{\prime}}\left\langle u_{i}\left[\mathbf{X}\left(t^{\prime}\right), t^{\prime}\right] u_{i}\left[\mathbf{X}\left(t^{\prime \prime}\right), t^{\prime \prime}\right]\right\rangle_{D X_{0}} d t^{\prime \prime} \\
& +2 \int_{t_{0}}^{t}\left\langle\left[X_{i}\left(t_{0}\right)-\left\langle X_{i}\left(t_{0}\right)\right\rangle_{D X_{0}}\right] u_{i}\left[\mathbf{X}\left(t^{\prime}\right)\right]\right\rangle_{D X_{0}} d t^{\prime} .
\end{aligned}
$$

The relation [Eq. (15)] has also been derived directly from Eq. (14) by using the Itô formalism [18].

As follows from the comparison with the third term in Eq. (15), the contribution $s_{u, i j}$ [Eq. (11)] to the covariance [Eq. $(10)]$ is of the Taylor-Kubo type $[9,19]$, i.e., given by time integrals of the Lagrangian velocity correlation. The novelty of this result is that Eq. (11) is a Taylor-Kubo relation valid for a deterministic velocity field or for a fixed realization of a random field. The contribution [Eq. (11)] of the variable velocity field is an equivalent expression of the usual term, given by a time integral of the correlation position velocity [see Eq. (A3)], derived from computations of the spatial moments of the concentration field [20] or within the Lagrangian approach used in this paper (e.g., [21]). Note that in previous works Taylor-Kubo contributions to the covariance of the advection-diffusion process or to the effective diffusion coefficients were obtained after averaging over ensembles of velocity realizations $[9,19,22,24]$ or as first-order approximations in velocity variance $[20,21,23,24]$.

The last term of Eq. (15) shows that Eq. (12) is the correlation of the Lagrangian velocity with the initial position cumulated in time. Hence $m_{i i}$ describes the memory of the diffusing particles. Further, using Eq. (14), we rewrite Eq. (15) as

$$
s_{i i}\left(t, t_{0}\right)=s_{i i}\left(t_{0}\right)+\tilde{s}_{i i}\left(t, t_{0}\right)+m_{i i}\left(t, t_{0}\right),
$$

where the sum of second and third terms of Eq. (15) is expressed in terms of displacements $\tilde{X}_{i}(t)=X_{i}(t)-X_{i}\left(t_{0}\right)$,

$$
\tilde{s}_{i i}\left(t, t_{0}\right)=\left\langle\left[\tilde{X}_{i}(t)-\left\langle\tilde{X}_{i}(t)\right\rangle_{D X_{0}}\right]^{2}\right\rangle_{D X_{0}} .
$$

Hence, Eq. (17) is the dispersion of the random variable $\tilde{X}_{i}$. The memory terms $m_{i i}$ can be equivalently expressed as correlations between displacements and initial positions of particles,

$$
m_{i i}\left(t, t_{0}\right)=2\left\langle\left[X_{i}\left(t_{0}\right)-\left\langle X_{i}\left(t_{0}\right)\right\rangle_{D X_{0}}\right]\left[\tilde{X}_{i}(t)-\left\langle\tilde{X}_{i}(t)\right\rangle_{D X_{0}}\right]\right\rangle_{D X_{0}} .
$$

Thus the variance [Eq. (16)] consists of a sum of two dispersion terms, $s_{i i}\left(t_{0}\right)+\widetilde{s}_{i i}\left(t, t_{0}\right)$, and a memory term, $m_{i i}\left(t, t_{0}\right)$.

Though Eqs. (16)-(18) were introduced here by using the equivalence of the Fokker-Plank and Itô descriptions for diffusion processes, it is noteworthy to mention that they are general relations that hold true for any continuous or discrete time-space process [25]. In fact, Eq. (16) is strictly equivalent to the usual definition of the variance, $s_{i i}=\left\langle\left[X_{i}\right.\right.$ $\left.\left.-\left\langle X_{i}\right\rangle_{D X_{0}}\right]^{2}\right\rangle_{D X_{0}}$. For deterministic processes, Eq. (16) without averages with subscript $D$ is a decomposition of the moment of inertia $s_{i i}$ of the cloud of particles moving on known trajectories $X_{i}(t)$, for instance, the trajectories of the dynamical system generated by a nonsingular velocity field [26].

\section{MEMORY TERMS FOR RANDOM INITIAL CONDITIONS}

Let us consider a process $\mathbf{X}(t)$, starting at $t_{0}$ from $\mathbf{X}\left(t_{0}\right)$ =0. Since $s_{i i}\left(t_{0}\right)$ and $m_{i i}\left(t, t_{0}\right)$ [Eq. (18)] vanish, Eq. (16) reduces to $s_{i i}\left(t, t_{0}\right)=\tilde{s}_{i i}\left(t, t_{0}\right)$, which is the dispersion of the random variable $\tilde{X}_{i}(t)=X_{i}(t)$. If one observes the same process from time $\tau>t_{0}$ on, then the initial positions are random variables $X_{i}(\tau)$, outcome of the evolution of the process for $t<\tau$. In these conditions, the dispersion of the total displacements $X_{i}(t)$ can be written according to Eq. (16) as

$$
\tilde{s}_{i i}\left(t, t_{0}\right)=\tilde{s}_{i i}\left(\tau, t_{0}\right)+\tilde{s}_{i i}(t, \tau)+m_{i i}(t, \tau),
$$

where the terms $\tilde{s}_{i i}$ are dispersions given by Eq. (17) for $\tilde{X}_{i}$ equal to $X_{i}(t), X_{i}(\tau)$, and $X_{i}(t)-X_{i}(\tau)$, respectively, and the memory term describes the correlation of the successive displacements,

$$
m_{i i}(t, \tau)=2\left\langle\left[\tilde{X}_{i}(\tau)-\left\langle\tilde{X}_{i}\right\rangle_{D X_{0}}(\tau)\right]\left[\tilde{X}_{i}(t)-\left\langle\tilde{X}_{i}\right\rangle_{D X_{0}}(t)\right]\right\rangle_{D X_{0}} .
$$

According to Eq. (19), cancellation of memory terms $m_{i i}(t, \tau)$ is equivalent to additivity of the dispersion $\widetilde{s}_{i i}$ with respect to nonoverlapping time intervals, $\widetilde{s}_{i i}\left(t, t_{0}\right)=\tilde{s}_{i i}\left(\tau, t_{0}\right)$ $+\tilde{s}_{i i}(t, \tau)$. In particular, if the dispersion of the particle displacements is a linear function of time, then the memory terms necessarily vanish. This is obviously the case of the Wiener process starting at $(0,0)$, for which the memory term [Eq. (20)] vanishes because the increments of the process are independent, $\left\langle\left[W_{i}(\tau)-W_{i}(0)\right]\left[W_{i}(t)-W_{i}(\tau)\right]\right\rangle_{D}=0$.

\section{A. Discrete diffusion processes with finite memory}

The following example illustrates the case of processes with finite memory which after a transient time reaches a diffusive regime characterized by linearity of the dispersion with respect to time.

The trajectory of the Wiener process can be simulated numerically by summing up Gaussian random variables, $Z$ $=\left\{Z_{n}, n=0, \pm 1, \pm 2, \ldots\right\}$, of mean zero and unit variance. This is the particular case $\phi=1$ of the more general algo- 
rithm for autoregressive processes of order 1 generated by the recursive relation $X_{n}=\phi X_{n-1}+Z_{n}$. For $0<\phi<1$, the discrete process $X=\left\{X_{n}\right\}$ is stationary, with mean zero, constant variance $s_{X}=1 /\left(1-\phi^{2}\right)$, and autocovariance $\left\langle X_{n} X_{n+r}\right\rangle=s_{X} \phi^{r}$. Finite sequences are also stationary, with mean zero and with the same covariance, if the first term $X_{0}$ is chosen as a random variable with the same variance $s_{X}$ as the infinite autoregressive processes [27]. Summing up realizations of $X_{n}$ is a simple way to obtain diffusion processes with memory. The process $\left\{Y_{n}, n>0\right\}$ starting from $Y_{0}=0$ generated by

$$
Y_{n}=Y_{n-1}+X_{n}=\sum_{s=1}^{n} X_{s}
$$

has the expectation $\left\langle Y_{n}\right\rangle=0$ and, because $\left\{X_{n}\right\}$ is stationary, the variance $s_{Y_{n}}=\left\langle Y_{n}^{2}\right\rangle$ can be expressed by a discrete TaylorKubo relation,

$$
s_{Y_{n}}=\sum_{s=1}^{n}\left\langle X_{s}^{2}\right\rangle+2 \sum_{r=1}^{n-1} \sum_{s=1}^{n-r}\left\langle X_{s} X_{s+r}\right\rangle=n s_{X}+2 s_{X} \sum_{r=1}^{n-1}(n-r) \phi^{r} .
$$

Einstein formula gives a finite diffusion coefficient [28],

$$
D=\lim _{n \rightarrow \infty} \frac{s_{Y_{n}}}{2 n}=\frac{s_{X}}{2}\left(1+2 \frac{\phi}{1-\phi}\right) .
$$

The discrete form of Eq. (19) is $\tilde{s}_{Y_{n}}=\tilde{s}_{Y_{l}}+\tilde{s}_{Y_{n, l}}+m_{Y_{n, l}}$, where $\tilde{s}_{Y_{n}}=s_{Y_{n}}$ whereas $\tilde{s}_{Y_{l}}$ and $\tilde{s}_{Y_{n, l}}$ are expressed analogous to Eq. (21) after replacing the upper summation limit by $l$ and the lower limit by $l+1$, respectively. It is ready to check that, for fixed $l, \lim _{n \rightarrow \infty}\left(\widetilde{s}_{Y_{l}}+\tilde{s}_{Y_{n, l}}\right) /(2 n)=D$; that is, the memory term $m_{Y_{n, l}}$ has no contribution to the diffusion coefficient [Eq. (22)]. This can also be checked directly by computing the memory term

$$
m_{Y_{n, l}}=2\left\langle\left(Y_{l}-Y_{0}\right)\left(Y_{n}-Y_{l}\right)\right\rangle=2 l s_{X} \sum_{r=l}^{n-1} \phi^{r}=2 l s_{X} \frac{1-\phi^{n-l}}{1-\phi} \phi^{l} .
$$

Since the limit $n \rightarrow \infty$ of Eq. (23) is a constant, $2 l s_{X} \phi^{l}$ $(1-\phi)$, the term $m_{Y_{n, l}} /(2 n)$ has no contribution to $D$. Moreover, the limit $n, l \rightarrow \infty, n>l$ of Eq. (23) vanishes, hence the increments of the diffusion process with memory $\left\{Y_{n}, n\right.$ $>0\}$ become independent in the long-time limit.

\section{B. Diffusion in random velocity fields}

If the velocity is a realization of a random space function, a key issue is whether the average over the ensemble of velocity realizations of the diffusion process described by Eqs. (2) and (14) behaves diffusively at some large time scale $[19,22,29]$. The relevant variance is now $\left\langle\left[X_{i}\right.\right.$ $\left.\left.-\left\langle X_{i}\right\rangle_{D X_{0} V}\right]^{2}\right\rangle_{D X_{0} V}$, which, as follows from Eq. (B1), is the second, central, spatial moment of the ensemble average concentration $\langle c(\mathbf{x}, t)\rangle_{V}=\langle\delta[\mathbf{x}-\mathbf{X}(t)]\rangle_{D X_{0} V}$. (Here and in the following, angular brackets with subscript $V$ denote ensemble averages.) The identity [Eq. (19)] can be used in investigations on the second moment of the mean concentra- tion when averages $\langle\cdots\rangle_{D X_{0}}$ are replaced by $\langle\cdots\rangle_{D X_{0} V}$. Further, let us consider the Itô process,

$$
X_{i}(t)=X_{i}(\tau)+\int_{\tau}^{t} V_{i}\left[\mathbf{X}\left(t^{\prime}\right)\right] d t^{\prime}+W_{i}(t-\tau),
$$

with the same coefficients and with initial position given by the solution $X_{i}(\tau)$ of the Eq. (14) at the moment $\tau$. Assuming that the Itô equation has unique solutions, from Eqs. (14) and (24) one obtains the following explicit dependence of the memory term [Eq. (20)] on the Lagrangian correlation function $C^{L}$ :

$$
m_{i i}(t, \tau)=2 \int_{\tau}^{t} \int_{0}^{\tau} C_{i i}^{L}\left(t^{\prime}, t^{\prime \prime}\right) d t^{\prime} d t^{\prime \prime}
$$

where

$$
\begin{aligned}
C_{i i}^{L}\left(t^{\prime}, t^{\prime \prime}\right)= & \left\langle V_{i}\left[\mathbf{X}\left(t^{\prime}\right), t^{\prime}\right] V_{i}\left[\mathbf{X}\left(t^{\prime \prime}\right), t^{\prime \prime}\right]\right\rangle_{D X_{0} V} \\
& -\left\langle V_{i}\left[\mathbf{X}\left(t^{\prime}\right), t^{\prime}\right]\right\rangle_{D X_{0} V}\left\langle V_{i}\left[\mathbf{X}\left(t^{\prime \prime}\right), t^{\prime \prime}\right]\right\rangle_{D X_{0} V} .
\end{aligned}
$$

While the question whether the Lagrangian correlation inherits properties of the Eulerian correlation of the random space function $\mathbf{V}(\mathbf{x})$ has no simple answer and requires proving specific limit theorems [19,22], some insight can however be provided by formal asymptotic expansions [29]. The simplest approach is that considering statistically homogeneous velocity fields and first-order approximations of the transport equations. For advection dominated transport problems, a consistent first-order approximation to the solutions of Itô equation [Eq. (14)] with initial condition $\mathbf{X}\left(t_{0}\right)=\mathbf{0}$ is obtained by the first iteration about the unperturbed solution $X_{i}^{(0)}(t)=\delta_{i, 1} U t$, where $U$ is the constant mean of the velocity field $\langle\mathbf{V}(\mathbf{x})\rangle_{V}$, assumed to be oriented along the one axis of the coordinate system: $X_{i}^{(1)}(t)=\int_{t_{0}}^{t} V_{i}\left(U t^{\prime}\right) d t^{\prime}+W_{i}\left(t-t_{0}\right)$ $[18,23,24]$. In this approximation the arguments of $V_{i}$ in Eq. (26) have to be replaced by $U t^{\prime}$ and $U t^{\prime \prime}$ and the Lagrangian correlation will be $C_{i i}^{L}\left(t^{\prime}, t^{\prime \prime}\right)=C_{i i}^{E}\left[U\left(t^{\prime}-t^{\prime \prime}\right)\right]$, where $C_{i i}^{E}(\mathbf{r})$ $=\left\langle V_{i}(\mathbf{x}) V_{i}(\mathbf{x}+\mathbf{r})\right\rangle_{V}-\left\langle V_{i}(\mathbf{x})\right\rangle_{V}\left\langle V_{i}(\mathbf{x}+\mathbf{r})\right\rangle_{V}$ is the homogeneous Eulerian correlation. The first-order approximation of the dispersion $\tilde{s}_{i i}\left(t, t_{0}\right)$, associated to the ensemble average concentration, becomes

$$
\tilde{s}_{i i}\left(t, t_{0}\right)=2 D t+2 \int_{t_{0}}^{t} d t^{\prime} \int_{t_{0}}^{t^{\prime}} C_{i i}^{E}\left[U\left(t^{\prime}-t^{\prime \prime}\right)\right] d t^{\prime \prime} .
$$

If the Eulerian field has finite correlation lengths $\lambda_{i i}$ $=1 /\left[C_{i i}^{E}(\mathbf{0})\right] \int C_{i i}^{E}(\mathbf{r}) d \mathbf{r}$, then the first-order approximation of the Lagrangian field also has finite correlation times $\lambda_{i i} / U$ [24]. Heuristically, because the velocity field has finite correlation scale, one assumes that the displacements over times larger than the correlation time will be uncorrelated and by the central limit theorem the process of diffusion in random fields behaves like a Gaussian diffusion (e.g., the macrodispersion model for transport of solutes in geological porous formations [30]). Nevertheless, rigorous proofs of this statement are only obtained under stronger assumptions on the velocity field $[19,22]$.

Following a common approach in physics literature $[14,31]$, the behavior of the process at large distances can be 
described by considering white noise correlations $C_{i i}^{E}$ $=Y_{i i} \delta(\mathbf{r})$. Then, the memory term [Eq. (25)] can be computed exactly and yields $m_{i i}(t, \tau)=0$. Similarly, from Eq. (27) one obtains the linear dispersion $\tilde{s}_{i i}\left(t, t_{0}\right)=2\left[D+Y_{i i} / U\right](t$ $\left.-t_{0}\right)$. Thus, for white noise correlations the memory terms vanish at all times, the displacement increments from Eq. (20) are uncorrelated, like in the case of a Gaussian diffusion, and the dispersion [Eq. (19)] is a linear-time function.

In case of finite correlation lengths, as for instance the often used isotropic exponential $C_{i i}^{L}=C_{i i}^{E}(0) e^{-\left(t^{\prime}-t^{\prime \prime}\right) U / \lambda}$ and Gaussian $C_{i i}^{L}=C_{i i}^{E}(0) e^{-\left(t^{\prime}-t^{\prime \prime}\right)^{2} U / \lambda}$ correlations, information on the asymptotic behavior can be obtained by considering the long-time limit of the time derivatives of the dispersion and memory terms. From Eq. (25) one obtains

$$
\lim _{t \rightarrow \infty} \frac{d}{d t} m_{i i}(t, \tau)=2 \lim _{t \rightarrow \infty} \int_{0}^{\tau} C_{i i}^{L}\left(t-t^{\prime \prime}\right) d t^{\prime \prime}=0
$$

because for $\left(t-t^{\prime \prime}\right) U \gg \lambda$ the Lagrangian correlation $C^{L}$ will be very close to zero. The derivative of the dispersion [Eq. (27)] instead approaches a finite limit and defines upscaled diffusion coefficients

$$
\begin{aligned}
D_{i i}^{*}=\frac{1}{2} \lim _{t \rightarrow \infty} \frac{d}{d t} \widetilde{s}_{i i}\left(t, t_{0}\right) & =D+\lim _{t \rightarrow \infty} \int_{t_{0}}^{t} C_{i i}^{E}\left[U\left(t-t^{\prime \prime}\right)\right] d t^{\prime \prime} \\
& =D+\frac{\lambda C^{E}(0)}{U} .
\end{aligned}
$$

Since, according to Eq. (28), the dispersion $\widetilde{s}_{i i}$ has a longtime-linear behavior, Eq. (19) implies the cancellation of the memory terms $m_{i i}$. It follows that for sufficiently fast decay of the Lagrangian correlations, the diffusing particles forget the memory and behave diffusively like Brownian particles.

A special situation, for which no approximations are required, is that of a system of particles undergoing diffusion in a stratified velocity field. If the advective velocities of the particles have only longitudinal components which depend randomly on the transverse coordinate alone and a white noise correlation, then the Lagrangian correlation [Eq. (26)] can be computed exactly: $C_{11}^{L}=C_{11}^{E}(0) e^{\left(t^{\prime}-t^{\prime \prime}\right)^{-1 / 2}} /(2 \sqrt{\pi D})$ $[14,31]$. Since the integral of $C_{11}^{L}$ diverges, the longitudinal Lagrangian velocity has an infinite correlation time and the decorrelation of the displacements from Eq. (20) cannot be expected. The longitudinal memory term computed from Eq. $(25)$

$$
m_{11}(t, \tau)=\frac{2 C_{11}^{E}(0)}{3 \sqrt{\pi D}}\left[\left(t-t_{0}\right)^{3 / 2}-\left(\tau-t_{0}\right)^{3 / 2}-(t-\tau)^{3 / 2}\right],
$$

expresses, according to Eq. (19), the nonlinearity of the dispersion terms with a $t^{3 / 2}$ time behavior [obtained explicitly by replacing $C_{11}^{L}$ in Eq. $\left.(27)\right], m_{11}(t, \tau)=\tilde{s}_{i i}\left(t, t_{0}\right)-\left[\tilde{s}_{i i}\left(\tau, t_{0}\right)\right.$ $\left.+\widetilde{s}_{i i}(t, \tau)\right]$. The memory term [Eq. (29)] is nonvanishing at all times $t>\tau$ and tends to infinity for $t \rightarrow \infty$. This shows that, according to Eq. (20), the particles undergoing diffusion in perfectly stratified velocity fields never forget the initial random position $X_{1}(\tau)$. Or equivalently, according to Eq. (25), the particles always remember the past Lagrangian velocity they had before the initial observation time $\tau$.

\section{MEMORY EFFECTS FOR DETERMINISTIC INITIAL CONDITIONS}

Now, we consider the memory terms [Eq. (18)] for initial positions $X_{i}\left(t_{0}\right)$ that are no longer the outcome of the evolution of the same process but arbitrary deterministic quantities. Since in this case the correlation of increments in Eq. (18) cannot be expressed by correlations of the Lagrangian velocity, the issue was investigated through numerical experiments.

Simulations of diffusion of large collections of particles in realizations of a random velocity field were carried out with the global random walk (GRW) algorithm [32]. Though equivalent with a superposition of many particle tracking procedures (Euler schemes for the Itô equation [Eq. (14)]), GRW is rather a cellular automaton: at given time step all the particles located at grid points are simultaneously advected with the local velocity and spread according to the random walk rule. This allows global simulations of diffusion for huge numbers of particles which render the statistical fluctuations of the estimated expectations $\langle\cdots\rangle_{D X_{0}}$ (e.g., concentration moments) smaller than the limit of double precision of the computing platform. For the simulations presented in the following this precision was ensured by using $10^{10}$ particles.

We considered a hydrological problem of contaminant transport through an isotropic two-dimensional aquifer system, characterized by logarithmic-normal distributed hydraulic conductivity $K$ with small variance $\sigma_{\ln K}^{2}=0.1$ and exponentially decaying isotropic correlation with correlation length $\lambda=1 \mathrm{~m}$. Darcy velocity fields were approximated numerically by Gaussian fields $[11,23]$. For fixed mean flow velocity $U=1 \mathrm{~m} / \mathrm{d}$ and isotropic local dispersion with constant coefficient $D=0.01 \mathrm{~m}^{2} / \mathrm{d}$, the Péclet number got a typical value $\mathrm{Pe}=U \lambda / D=100$. Details on algorithm and numerical setup can be found in Ref. [33].

\section{A. Memory terms for fixed velocity realizations}

To estimate memory terms [Eq. (18)] for a single realization we considered a number of 121 initial positions $\mathbf{X}\left(t_{0}\right)$ uniformly distributed in rectangular domains with dimensions $L_{1} \lambda \times L_{2} \lambda$. By releasing $10^{10}$ particles from each initial position, we performed GRW simulations to compute the displacements along the trajectories of the diffusion processes starting from these positions. Finally, the correlations between displacements and initial positions from Eq. (18) were computed by averages over the initial positions $\mathbf{X}\left(t_{0}\right)$. The results presented in Fig. 1 show that at early times the memory terms $m_{i i}$ increase with the dimension of the source in the $i$ direction and are mainly significant for asymmetric sources.

The overall decay of $m_{i i}$ in all cases indicates that the averages $\left\langle u_{i}[\mathbf{X}(t)]\right\rangle_{D}$ of the fluctuations of the Lagrangian velocity averaged over realizations of the diffusion process for fixed initial positions $X_{i}\left(t_{0}\right)$ become independent of $X_{i}\left(t_{0}\right)$ [see Eq. (15)]. This can be the case if $\left\langle u_{i}[\mathbf{X}(t)]\right\rangle_{D}$ tends to the 

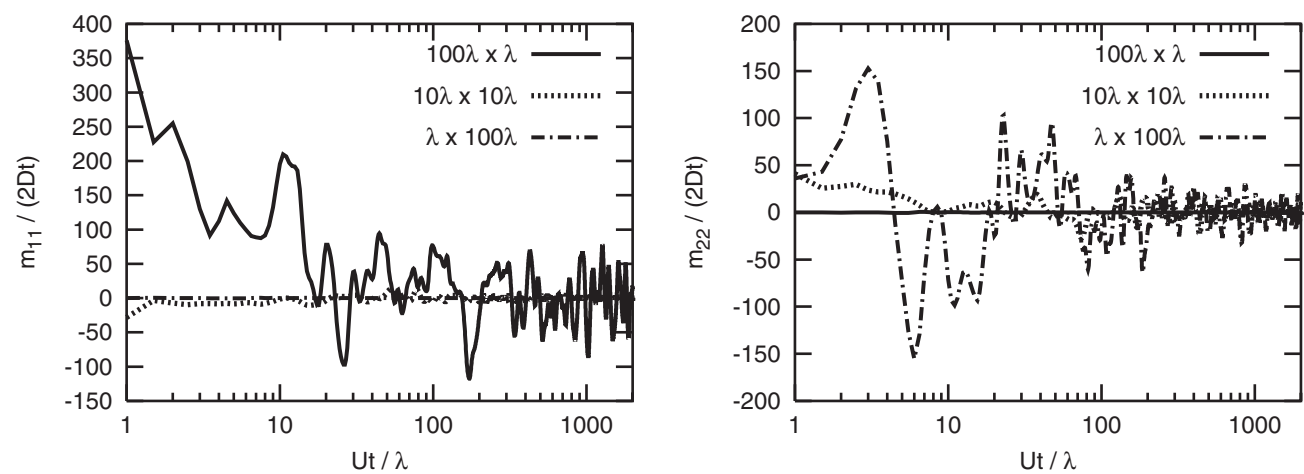

FIG. 1. Memory terms in longitudinal (left) and transverse (right) directions with respect to the mean flow obtained from GRW simulations for a given velocity field and for sources with different dimensions, shapes, and orientations.

constant ensemble averages of the Eulerian field. Although such self-averaging properties can only be proved in particular cases (e.g., [10]), they are often found in numerical modeling of transport in random fields with finite correlation scales $[11,25]$. In our case, the self-averaging is indicated by the decay in time of the fluctuations of $\left\langle u_{i}[\mathbf{X}(t)]\right\rangle_{D}$ and the good agreement between the space-averaged Lagrangian velocity and the ensemble mean Eulerian velocity shown in Fig. 2. The two panels of Fig. 2 also show that the shape of the initial distribution (longitudinal and transverse slabs of thickness $\lambda$ ) has little influence on the self-averaging behavior, provided that the support of the initial concentration extends over at least one correlation length in all directions.

Intriguingly, in the case of diffusion in perfectly stratified flows with infinite correlation range and infinitely persistent memory of the random initial conditions, analyzed in Sec. III B, the dependence on deterministic initial conditions induces only transitory effects. The transverse dispersion is that of a memory-free Brownian motion with $m_{22}=0$ and, because the longitudinal velocity does not depend on the longitudinal coordinates, $m_{11}$ [Eq. (18)] identically vanishes at all times. Equation (12) shows that the off-diagonal term $m_{21}$, corresponding to the correlation between longitudinal velocities and transverse initial positions of particles, $\left\langle X_{2}\left(t_{0}\right)\left\langle u_{1}\left[X_{2}(t)\right]\right\rangle_{D}\right\rangle_{D X_{0}}$, is nonvanishing at finite times. However, since $u_{1}$ has a finite correlation length in the transverse direction it has a self-averaging behavior similar to that in Fig. 1 and $m_{21}$ vanishes in the long-time limit.

Longitudinal slab $100 \lambda \times \lambda$

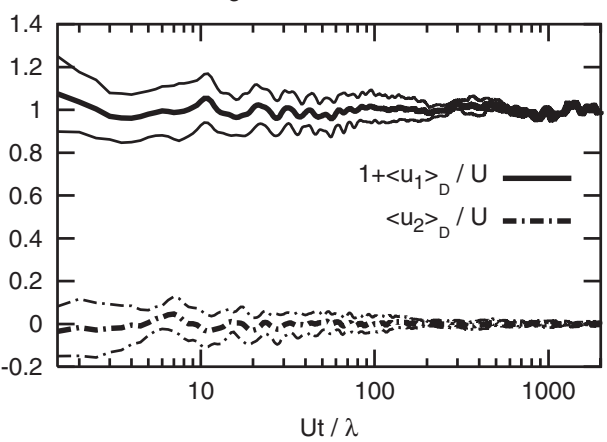

\section{B. Persistent memory of the initial conditions}

When diffusion takes place in random environments, the ensemble averaged variance of the process and the second moment of the ensemble averaged concentration have different behaviors and describe different features of the physical process $[14,15,34]$. Their difference is the variance of the center of mass, which is also strongly influenced by the initial conditions of the transport problem $[25,33]$.

To account for the randomness of the center of mass, we define dispersion terms

$$
\begin{array}{r}
\sigma_{i i}=\left\langle\left[X_{i}-\left\langle X_{i}\right\rangle_{D X_{0} V}\right]^{2}\right\rangle_{D X_{0}}, \\
r_{i i}=\left[\left\langle X_{i}\right\rangle_{D X_{0}}-\left\langle X_{i}\right\rangle_{D X_{0} V}\right]^{2}
\end{array}
$$

and we write the variance [Eq. (16)] in the equivalent form

$$
s_{i i}=\sigma_{i i}-r_{i i} .
$$

For processes governed by the Fokker-Planck equation [Eq. (2)] and Itô equation [Eq. (14)], Eq. (32) is obtained by replacing in the variance [Eq. (10)] the center of mass for a single realization $\left\langle X_{i}\right\rangle_{D X_{0}}=\mu_{i}\left(t, t_{0}\right)=\mu_{i}\left(t_{0}\right)+\int_{t_{0}}^{t^{\prime}} \bar{V}_{i}\left(t^{\prime}\right) d t^{\prime}$ with its ensemble average $\mu_{i}\left(t_{0}\right)+\int_{t_{0}}^{t^{\prime}}\left\langle\bar{V}_{j}(t)\right\rangle_{V} d t^{\prime}$ and by subtracting the correction [Eq. (31)]. The latter is expressed by using Eq. (9) as

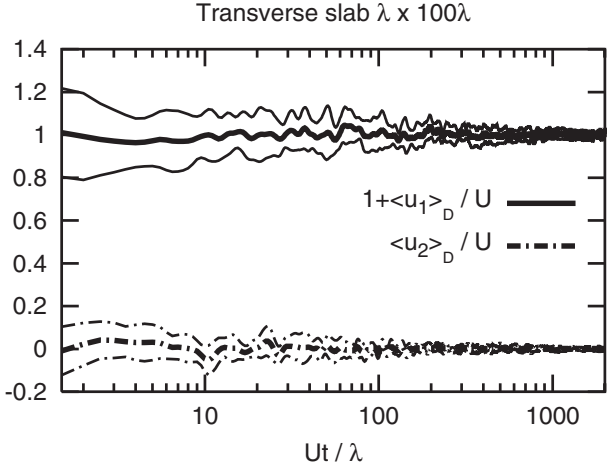

FIG. 2. Averages over 121 initial positions $\mathbf{X}\left(t_{0}\right)$ (thick lines) and standard deviations (thin lines) of the mean Lagrangian velocity $\left\langle u_{i}[\mathbf{X}(t)]\right\rangle_{D}$ for longitudinal and transverse slab sources. 


$$
\begin{aligned}
r_{i i}\left(t, t_{0}\right)= & \int_{t_{0}}^{t} d t^{\prime} \int \hat{u}_{i}\left(\mathbf{x}, t^{\prime}\right) c\left(\mathbf{x}, t^{\prime}\right) d \mathbf{x} \\
& \times \int_{t_{0}}^{t} d t^{\prime \prime} \int \hat{u}_{i}\left(\mathbf{x}^{\prime}, t^{\prime \prime}\right) c\left(\mathbf{x}^{\prime}, t^{\prime \prime}\right) d \mathbf{x}^{\prime},
\end{aligned}
$$

where the velocity fluctuation is now defined with respect to the ensemble averaged velocity, $\hat{u}_{i}(\mathbf{x}, t)=V_{i}(\mathbf{x}, t)-\left\langle\bar{V}_{i}(t)\right\rangle_{V}$. Expression (10) can thus be rewritten in the equivalent form,

$$
s_{i i}\left(t, t_{0}\right)=s_{i i}\left(t_{0}\right)+2 D\left(t-t_{0}\right)+s_{\hat{u}, i i}\left(t, t_{0}\right)+m_{i i}\left(t, t_{0}\right)-r_{i i}\left(t, t_{0}\right),
$$

where the memory term $m_{i i}\left(t, t_{0}\right)$ is given by Eq. (12) for $i$ $=j$ and the contribution of the velocity fluctuations $\hat{u}_{i}$ has a form similar to Eq. (11),

$$
\begin{aligned}
s_{\hat{u}, i i}\left(t, t_{0}\right)= & \int_{t_{0}}^{t} d t^{\prime} \int_{t_{0}}^{t^{\prime}} d t^{\prime \prime} \int c\left(\mathbf{x}_{0}, t_{0}\right) d \mathbf{x}_{0} \\
& \times \iint \hat{u}_{i}\left(\mathbf{x}^{\prime}, t^{\prime \prime}\right) \hat{u}_{i}\left(\mathbf{x}, t^{\prime}\right) g\left(\mathbf{x}, t \mid \mathbf{x}^{\prime}, t^{\prime}\right) \\
& \times g\left(\mathbf{x}^{\prime}, t^{\prime} \mid \mathbf{x}_{0}, t_{0}\right) d \mathbf{x} d \mathbf{x}^{\prime} .
\end{aligned}
$$

The ensemble average of the velocity correlation under the time integrals in Eq. (34) is the mean Lagrangian correlation [Eq. (26)] analyzed through first-order approximations in Sec. III B.

The ensemble average of Eq. (32) is a well known identity $[15,20,33,34]$ which relates the expected second moment $S_{i i}=\left\langle s_{i i}\right\rangle_{V}$ to the second moment of the mean concentration $\Sigma_{i i}=\left\langle\sigma_{i i}\right\rangle_{V}$ and the variance of the center of mass $R_{i i}=\left\langle r_{i i}\right\rangle_{V}$,

$$
S_{i i}=\Sigma_{i i}-R_{i i} \text {. }
$$

Assuming all necessary joint measurability conditions which allow permutations of averages [17] leads to

$$
\Sigma_{i i}=s_{i i}\left(t_{0}\right)+\left\langle X_{i i}\right\rangle_{X_{0}}+M_{i i}+Q_{i i},
$$

where $X_{i i}=\left\langle\left[\tilde{X}_{i}-\left\langle\tilde{X}_{i}\right\rangle_{D V}\right]^{2}\right\rangle_{D V}$ is the one-particle dispersion (defined by averaging with respect to $D$ and $V$ for a fixed initial position), $M_{i i}=\left\langle m_{i i}\right\rangle_{V}$ is the mean memory term, and $Q_{i i}=\left\langle\left[\left\langle\tilde{X}_{i}\right\rangle_{D V}-\left\langle\tilde{X}_{i}\right\rangle_{D X_{0} V}\right]^{2}\right\rangle_{X_{0}}$ is the spatial variance of the oneparticle center of mass $\left\langle\tilde{X}_{i}\right\rangle_{D V}$ (computed by averages over initial positions) [35].

The terms of Eq. (36) depend, via the trajectory [Eq. (14)], on the Lagrangian velocity field. If the Lagrangian field is statistically homogeneous the one-particle center of mass $\left\langle\widetilde{X}_{i}\right\rangle_{D V}$ and dispersion $X_{i i}$ are independent of $X_{0}[17,24]$; hence they are memory-free quantities. Then $M_{i i}$ and $Q_{i i}$ vanish and Eq. (36) takes on the simpler form,

$$
\Sigma_{i i}=s_{i i}\left(t_{0}\right)+X_{i i} \text {. }
$$

Assuming Lagrangian homogeneity leads, according to Eqs. (33) and (35), to $\Sigma_{i i}=\left\langle\sigma_{i i}\right\rangle_{V}=s_{i i}\left(t_{0}\right)+2 D\left(t-t_{0}\right)+\left\langle s_{\hat{u}, i i}\right\rangle_{V}$. Then, the upscaled diffusion coefficients describing the behavior of the ensemble mean concentration, $D_{i i}^{*}=\lim _{t \rightarrow \infty} \frac{1}{2 t} \Sigma_{i i}$ $=\frac{1}{2} \lim _{t \rightarrow \infty} \frac{d}{d t} \Sigma_{i i}$, are the sum between the local coefficient $D$ and the long-time limit of the half derivative of the ensemble averages $\left\langle s_{\hat{u}, i i}\right\rangle_{V}$ of the velocity fluctuation contributions [Eq. (34)]. Their first-order approximations [Eq. (28)] are related to the statistics of the hydraulic conductivity field by

$$
D_{i i}^{*}=D+\delta_{1 i} \sigma_{\ln K}^{2} U \lambda,
$$

which for the parameters of the numerical experiment presented here take the values $D_{11}^{*}=0.11 \mathrm{~m}^{2} / \mathrm{d}$ and $D_{22}^{*}$ $=0.01 \mathrm{~m}^{2} / \mathrm{d}[33]$.

The dispersion terms [Eqs. (30)-(32)] were estimated from GRW simulations by using for every velocity realization $10^{10}$ particles that were initially uniformly distributed in rectangular domains $L_{1} \lambda \times L_{2} \lambda$ or released from the origin of the computational grid. For each initial condition, 1024 realizations of the velocity field were used to asses ensemble averages $\langle\cdots\rangle_{V}$ (expectations and standard deviations of various concentration moments). Preliminary tests and comparison with reference simulations using an algorithm free of overshooting errors [23] showed that the overall precision of this Monte Carlo approach was of the order of the local dispersion $2 D t$ at early times and smaller than $D t$ after simulation times of about 30 dimensionless times $U t / \lambda$.

Figure 3 shows that for large dimensions of the support of the initial concentration (the source of particles) $S_{i i} \approx \Sigma_{i i}$. Hence, the variance of the center of mass $R_{i i} \approx 0$, a property which is quite insensitive to the shape and orientation of the source. This is a somewhat expected result because for transport in velocity fields with finite correlation range, which are ergodic [36], the center of mass for point sources $\left\langle X_{i}\right\rangle_{D}$ is ergodic too, i.e., the space average approximates the ensemble average, $\left\langle X_{i}\right\rangle_{D X_{0}} \approx\left\langle X_{i}\right\rangle_{D V}$, and according to Eq. (31) $R_{i i}=\left\langle r_{i i}\right\rangle_{D V} \approx 0$.

Another information provided by Fig. 3 is that for large slab sources transverse to the $i$ direction, for which the memory terms [Eq. (18)] are small [Fig. 1], $\Sigma_{i i}-s_{i i}\left(t_{0}\right)$ is independent of the source dimension. Therefore, we approximated the memory-free one-particle dispersion $X_{i i}$ by using in Eq. (37) the second moment of the mean concentration $\Sigma_{i i}$ estimated from GRW simulations done for the largest slab source $(L=100)$ oriented perpendicular to the $i$ axis. Since it has been found that the term $Q_{i i}$ is much smaller than the local dispersion 2Dt [35], the irregular behavior at early times of $\Sigma_{i i}$ for different initial conditions can be attributed, according to Eq. (36), to the mean memory terms $M_{i i}=\Sigma_{i i}$ $-s_{i i}\left(t_{0}\right)-X_{i i}$.

The mean memory terms are a consequence of the statistical inhomogeneity of the Lagrangian velocity field, for which the ensemble average of Eq. (12) is nonvanishing. Such memory effects on the ensemble averaged dispersion can be tracked back to the lack of smoothness of the velocity samples of the random field with exponential correlation used in simulations [24]. The issue is somewhat similar to that of memory-induced oscillations of the diffusion coefficient observed in the case of charged particles driven by a uniform magnetic field and a stationary Gaussian stochastic force with exponential time correlation [8], as well as in the case of particles driven by an anticorrelated autoregressive noise [28]. However, in the two latter examples the memory mechanism is no longer the dependence of the Lagrangian statistics on deterministic initial conditions but an intrinsic 

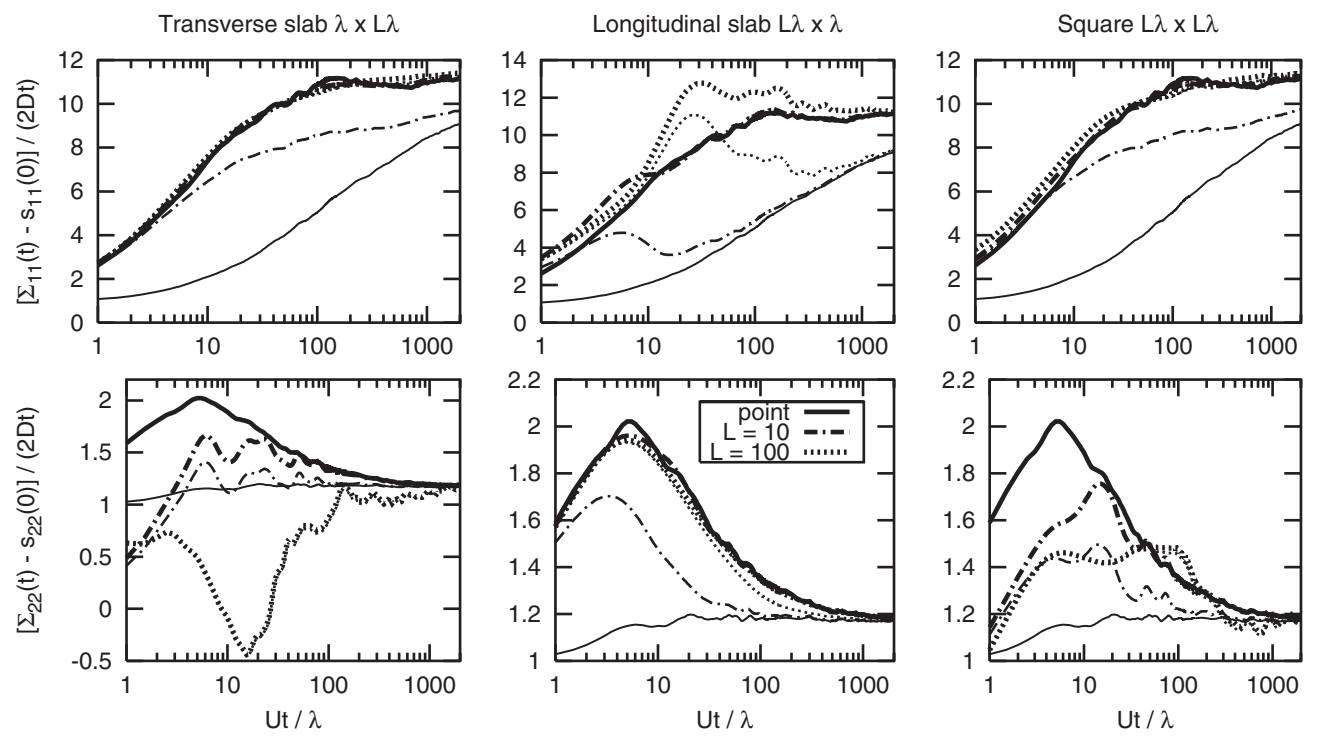

FIG. 3. Second moments of the ensemble average concentration $\Sigma_{i i}$ and ensemble averaged second moments $S_{i i}$ (thin lines) for different shapes and extensions of the source.

interdependence of the increments of the process due to the colored noise [28].

The smallness of the standard deviation $\operatorname{SD}\left(\sigma_{i i}\right)$ for large slab sources perpendicular to the $i$ direction shown in Fig. 4 indicates that $\sigma_{i i}-s_{i i}\left(t_{0}\right)$ also approximates $X_{i i}$ for such initial conditions. The large values of $\operatorname{SD}\left(\sigma_{i i}\right)$ for large slab sources parallel to the $i$ direction are due, according to Eq. (33), to the large memory terms $m_{i i}$ which, as shown by Eq. (18), increase with the source dimension. We have seen that for large sources, irrespective of their shape and orientation, $r_{i i}$ $\approx 0$. Thus, according to Eq. (32) $\sigma_{i i} \approx s_{i i}$ and we can adopt the following estimation of the memory terms,

$$
m_{i i}\left(t-t_{0}\right)=s_{i i}\left(t-t_{0}\right)-s_{i i}\left(t_{0}\right)-X_{i i}\left(t-t_{0}\right) .
$$

As seen in Fig. 3, the long-time limit of the effective diffusion coefficients, defined by the half slope of the mean dispersion $\left[S_{i i}-s_{i i}\left(t_{0}\right)\right] /\left(2\left(t-t_{0}\right)\right.$, approaches the upscaled coefficients [Eq. (38)]. The relevance of the upscaled coefficients for single realizations of the transport is an ergodicity issue. The overall trend of fluctuations shown in Fig. 4 indicates that single-realization dispersion coefficients are selfaveraging. Together, the results from Figs. 3 and 4 indicate an ergodic behavior in the sense that the mean square distance between single-realization and upscaled coefficients decreases in time [33].

Another ergodic behavior of interest in practical applications is that with respect to the memory-free dispersion $X_{i i}$, which is often available from estimations of the dispersion terms [Eq. (34)] for given correlations of the Eulerian veloc-
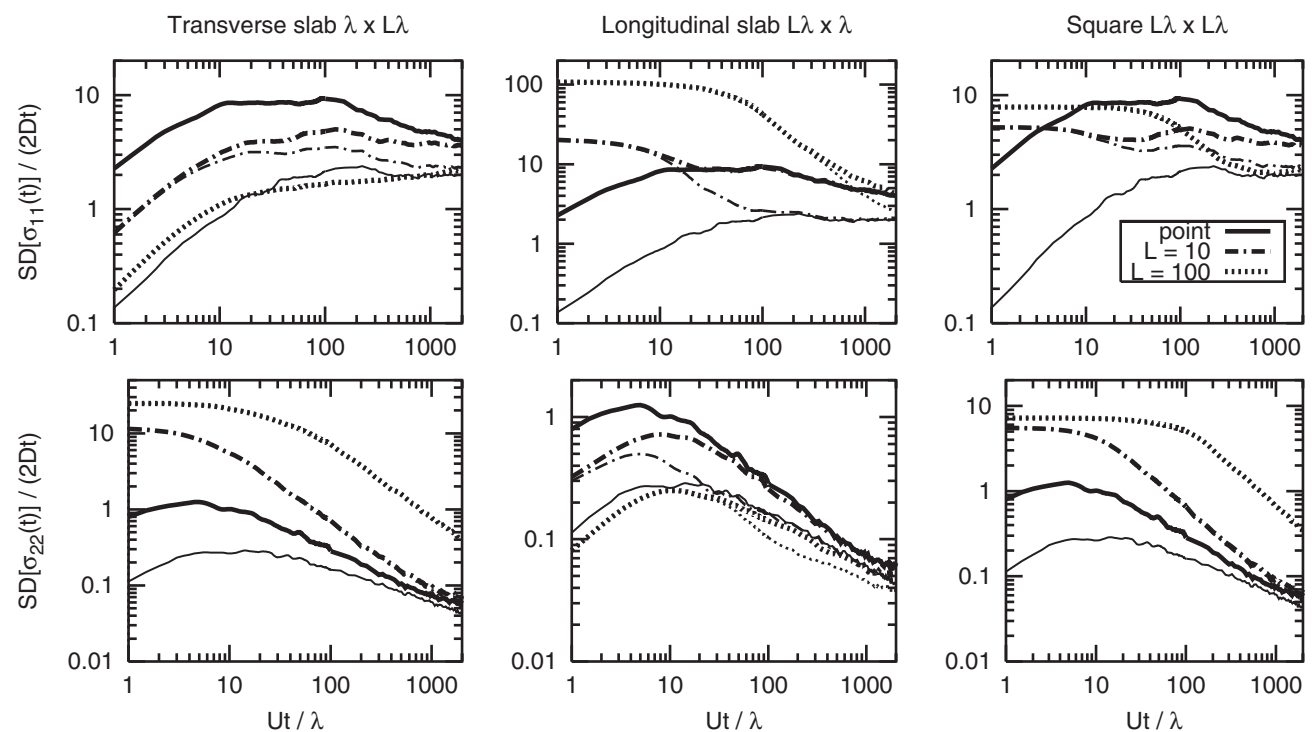

FIG. 4. Standard deviation of the second moment with respect to ensemble averaged center of mass $\sigma_{i i}$ and standard deviation of the second moment of the actual center of mass $s_{i i}$ (thin lines) for different shapes and extensions of the source. 

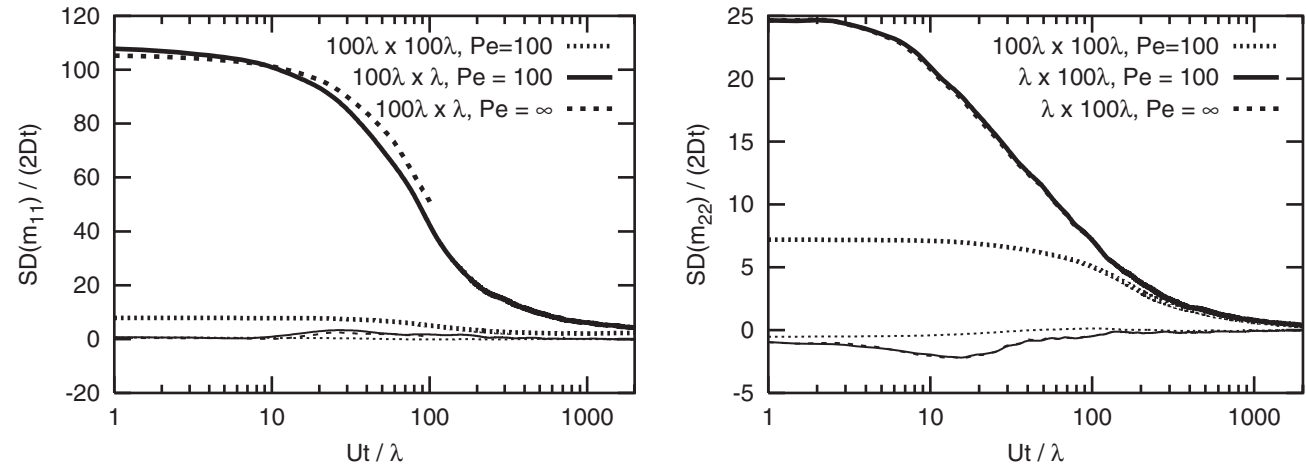

FIG. 5. Standard deviations of longitudinal (left) and transverse (right) memory terms and the corresponding mean values (thin lines).

ity field $[11,23]$. Using Eq. (39), we estimated the mean and the standard deviations of the largest memory terms $m_{i i}$ corresponding to sources with the largest extension in the $i$ direction. The results presented in Fig. 5 show that the mean memory terms are negligible small as compared to the standard deviations. So, practically the mean square distance between $s_{i i}$ and $X_{i i}$ is given by $\operatorname{SD}\left(m_{i i}\right)$. In Fig. 5 we also represented the memory terms for pure advective transport with $\mathrm{Pe}=\infty$, simulated by dropping the diffusion step in the GRW algorithm. It can be seen that the standard deviations are almost the same as in the case with $\mathrm{Pe}=100$. According to Eq. (39), the extinction of the memory terms is equivalent to the self-averaging of the single-realization variance $s_{i i}$ and of the effective diffusion coefficients. At finite times, memory effects manifest mainly through deviations of single-realization dispersion from the ideal model-behavior described by the memory-free dispersion $X_{i i}$. Such effects strongly depend on the shape, the orientation, and the spatial extension of the source of particles.

\section{CONCLUSIONS}

We decomposed the variance of the transport processes in dispersion and memory terms and for continuous diffusion process we derived explicit relations between these terms and the coefficients of the Fokker-Planck equation. Nevertheless, this decomposition is a general property of the variance and allows investigations on discrete processes as well.

The memory terms govern the preasymptotic behavior of the transport process. We have shown that normal diffusion occurs only if the memory terms vanish. This happens when the diffusing particles forget their past itinerary. For diffusion in statistically homogeneous velocity fields with finite correlation lengths, we found that for finite correlation times of the Lagrangian velocity or convergent Kubo formula, the particles lose the memory in the long-time limit and normal diffusion occurs. We found a similar behavior for a discrete diffusion process with finite memory generated by autoregressive noise.

Memory terms also quantify the persistent influence of the deterministic initial conditions on the behavior of the transport process. Large deterministic initial distributions of the cloud of particles cause large memory terms which prevent the use of the one-particle dispersion as a model for the preasymptotic transport regime. Numerical simulations of diffusion in space random velocity fields with finite correlation range indicated the extinction of the memory terms after considerably large times, corresponding to hundreds of correlation scales, as well as the self-averaging behavior of the variance and its tendency toward normal diffusion.

Thus, the issue of memory effects investigated in this paper can be partially answered: diffusing particles forget the memory of the deterministic initial position, as well as the memory of their past itinerary, when they evolve in timeindependent random environments with finite spatial correlation scales. Indefinitely persistent memory can be found, for instance, in case of diffusion in velocity fields with infinite spatial correlation range.

The behavior of the diffusion in time-variable environments requires further investigations. For instance, it is known that a sufficiently fast decay of the time correlation functions ensures the convergence toward a normal diffusion even in absence of spatial decorrelation [16,19]. However, for velocity fields with oscillating time correlations the condition of convergent Kubo formula, although a necessary condition for normal diffusion limit, may be far from being sufficient [19]. The challenge is to find the meaning of the memory terms for anomalous diffusion in conditions of nontrivial interplay of temporal and spatial correlations.

For continuous processes, analyses of memory effects via Lagrangian velocity correlations assume unique solutions of the Itô and Fokker-Planck equations. Nevertheless, the lack of smoothness of the velocity samples often precludes the existence of unique solutions. Moreover, as shown in Ref. [24], uniqueness is also an essential ingredient in proving the translation invariance of the ensemble average of the fundamental solution of the Fokker-Planck equation and the equivalent property of statistical homogeneity of the Lagrangian velocity field. When these properties are not ensured, as in case of our numerical setup, deterministic initial conditions induce memory effects on the effective diffusion coefficients. Even though in such cases first-order approximations still capture the asymptotic behavior [24], numerical models have to be developed for the transitory regime.

In this paper, memory effects were quantified in a straightforward way by correlations between the displacements of the particles and starting positions. In Ref. [37] it was shown that memory effects on diffusion coefficients are described in more detail by a hierarchy of Lagrangian corre- 
lations, sampled on increasing paths on the set of trajectories starting at a given initial position. A particular case is the expansion of the diffusion coefficients in sums of correlations of increments of the process sampled on a single trajectory $[28,38]$. Such representations of the diffusion coefficients, using double summations of correlations, suggest possible connections with the method of memory kernels $[2,4,5]$ and motivate further work.

\section{ACKNOWLEDGMENTS}

The research reported in this paper was supported by Deutsche Forschungsgemeinschaft Grant No. SU 415/1-2, Jülich Supercomputing Centre Project No. JICG41, and Romanian Ministry of Education and Research Grant No. 2-CEx06-11-96.

\section{APPENDIX A: MEAN VALUE AND COVARIANCE COMPONENTS}

The coefficients of the Fokker-Planck equation defined in Eqs. (5) and (6) can be related under the conditions formulated in Eqs. (4), (7), and (8) to the time derivatives of the mean and covariance components. Therefore, to derive the general expressions of the mean and covariances, we first compute their derivatives.

The derivative of the first moment can be computed as follows:

$$
\begin{aligned}
\frac{d}{d t} \mu_{i}(t)= & \lim _{\Delta t \rightarrow 0} \frac{1}{\Delta t}\left[\mu_{i}(t+\Delta t)-\mu_{i}(t)\right] \\
= & \lim _{\Delta t \rightarrow 0} \frac{1}{\Delta t}\left[\int x_{i}^{\prime} c\left(\mathbf{x}^{\prime}, t+\Delta t\right) d \mathbf{x}^{\prime}-\int x_{i} c(\mathbf{x}, t) d \mathbf{x}\right] \\
= & \int\left[\lim _{\Delta t \rightarrow 0} \frac{1}{\Delta t} \int\left(x_{i}^{\prime}-x_{i}\right) g\left(\mathbf{x}^{\prime}, t+\Delta t \mid \mathbf{x}, t\right) d \mathbf{x}^{\prime}\right] \\
& \times c(\mathbf{x}, t) d \mathbf{x},
\end{aligned}
$$

where we used Eq. (3) and the normalization property of $g$. For $\Delta t \rightarrow 0$, using Eqs. (4), (5), and (7) one obtains

$$
\frac{d}{d t} \mu_{i}(t)=\int V_{i}(\mathbf{x}, t) c(\mathbf{x}, t) d \mathbf{x}=\bar{V}_{i}(t) .
$$

By integrating Eq. (A1) we get Eq. (9) in the main text.

To compute the derivative of the variance we proceed like for the mean,

$$
\begin{aligned}
s_{i j}(t+\Delta t)-s_{i j}(t)= & \int x_{i}^{\prime} x_{j}^{\prime} c\left(\mathbf{x}^{\prime}, t+\Delta t\right) d \mathbf{x}^{\prime}-\int x_{i} x_{j} c(\mathbf{x}, t) d \mathbf{x} \\
& -\left[\mu_{i}(t+\Delta t) \mu_{j}(t+\Delta t)-\mu_{i}(t) \mu_{j}(t)\right] \\
= & \int c(\mathbf{x}, t) d \mathbf{x} \int\left(x_{i}^{\prime}-x_{i}\right)\left(x_{j}^{\prime}-x_{j}\right) g\left(\mathbf{x}^{\prime}, t\right. \\
& +\Delta t \mid \mathbf{x}, t) d \mathbf{x}^{\prime}+\int x_{i} c(\mathbf{x}, t) d \mathbf{x} \\
& \times \int\left(x_{j}^{\prime}-x_{j}\right) g\left(\mathbf{x}^{\prime}, t+\Delta t \mid \mathbf{x}, t\right) d \mathbf{x}^{\prime}
\end{aligned}
$$

$$
\begin{aligned}
& +\int x_{j} c(\mathbf{x}, t) d \mathbf{x} \int\left(x_{i}^{\prime}-x_{i}\right) g\left(\mathbf{x}^{\prime}, t\right. \\
& +\Delta t \mid \mathbf{x}, t) d \mathbf{x}^{\prime}-\left[\mu_{i}(t+\Delta t) \mu_{j}(t+\Delta t)\right. \\
& \left.-\mu_{i}(t) \mu_{j}(t)\right]
\end{aligned}
$$

and using Eqs. (4)-(8) for $\Delta t \rightarrow 0$, we obtain the derivative

$$
\begin{aligned}
\frac{d}{d t} s_{i j}(t)= & 2 \int D_{i j}(\mathbf{x}, t) c(\mathbf{x}, t) d \mathbf{x}+\int\left[x_{i} V_{j}(\mathbf{x}, t)\right. \\
& \left.+x_{j} V_{i}(\mathbf{x}, t)\right] c(\mathbf{x}, t) d \mathbf{x}-\frac{d}{d t}\left[\mu_{i}(t) \mu_{j}(t)\right],
\end{aligned}
$$

which after expressing the last term by Eq. (A1) takes the form

$$
\begin{aligned}
\frac{d}{d t} s_{i j}(t)= & 2 \int D_{i j}(\mathbf{x}, t) c(\mathbf{x}, t) d \mathbf{x}+\int\left\{x_{i}\left[V_{j}(\mathbf{x}, t)-\bar{V}_{j}(t)\right]\right. \\
& \left.+x_{j}\left[V_{i}(\mathbf{x}, t)-\bar{V}_{i}(t)\right]\right\} c(\mathbf{x}, t) d \mathbf{x} .
\end{aligned}
$$

Next, we highlight the dependence on the initial positions of the second term in Eq. (A2) and obtain an equivalent expression of the time derivative of $s_{i j}(t)$,

$$
\begin{aligned}
\frac{d}{d t} s_{i j}(t)= & 2 \int D_{i j}(\mathbf{x}, t) c(\mathbf{x}, t) d \mathbf{x}+\int c\left(\mathbf{x}_{0}, t_{0}\right) d \mathbf{x}_{0} \int\left\{\left(x_{i}-x_{0 i}\right)\right. \\
& \times\left[V_{j}(\mathbf{x}, t)-\bar{V}_{j}(t)\right]+\left(x_{j}-x_{0 j}\right)\left[V_{i}(\mathbf{x}, t)\right. \\
& \left.\left.-\bar{V}_{i}(t)\right]\right\} g\left(\mathbf{x}, t \mid \mathbf{x}_{0}, t_{0}\right) d \mathbf{x}+\int c\left(\mathbf{x}_{0}, t_{0}\right) d \mathbf{x}_{0} \\
& \times \int\left\{\left[x_{0 i}-\mu_{i}\left(t_{0}\right)\right]\left[V_{j}(\mathbf{x}, t)-\bar{V}_{j}(t)\right]+\left[x_{0 j}-\mu_{j}\left(t_{0}\right)\right]\right. \\
& \left.\times\left[V_{i}(\mathbf{x}, t)-\bar{V}_{i}(t)\right]\right\} g\left(\mathbf{x}, t \mid \mathbf{x}_{0}, t_{0}\right) d \mathbf{x} .
\end{aligned}
$$

To analyze the second term in Eq. (A3), we consider a time sequence $t_{0} \leq t_{1} \cdots<t_{k}<t_{k+1} \cdots \leq t_{n}=t, t_{k+1}-t_{k}=\Delta t$, and the joint probabilities $p$ of the sequence of events $\left(\mathbf{x}_{0}, t_{0}\right), \ldots,\left(\mathbf{x}_{n-1}, t_{n-1}\right),(\mathbf{x}, t)$. The contribution of $\left(x_{i}\right.$ $\left.-x_{0 i}\right) V_{j}(\mathbf{x}, t)$ can be expressed using the ChapmanKolmogorov equation and the consistency property of $p$ that integrating over intermediate states one obtains reduced order joint probabilities as follows:

$$
\begin{aligned}
\int c & \left(\mathbf{x}_{0}, t_{0}\right) d \mathbf{x}_{0} \int\left(x_{i}-x_{0 i}\right) V_{j}(\mathbf{x}, t) g\left(\mathbf{x}, t \mid \mathbf{x}_{0}, t_{0}\right) d \mathbf{x} \\
= & \int \ldots \int\left[\sum_{k=0}^{n-1}\left(x_{k+1, i}-x_{k, i}\right)\right] V_{j}(\mathbf{x}, t) \\
& \times p\left(\mathbf{x}, t ; \mathbf{x}_{n-1}, t_{n-1} ; \ldots ; \mathbf{x}_{0}, t_{0}\right) d \mathbf{x} d \mathbf{x}_{n-1} \ldots d \mathbf{x}_{0} \\
= & \sum_{k=0}^{n-1} \iiint\left(x_{k+1, i}-x_{k, i}\right) V_{j}(\mathbf{x}, t) \\
& \times g\left(\mathbf{x}, t \mid \mathbf{x}_{k+1}, t_{k+1}\right) g\left(\mathbf{x}_{k+1}, t_{k+1} \mid \mathbf{x}_{k}, t_{k}\right) c\left(\mathbf{x}_{k}, t_{k}\right) d \mathbf{x} d \mathbf{x}_{k+1} d \mathbf{x}_{k} \\
& x_{n-1} \int c\left(\mathbf{x}_{k}, t_{k}\right) d \mathbf{x}_{k} \int\left(x_{k+1, i}-x_{k, i}\right) \\
= & \sum_{k=0} \int
\end{aligned}
$$




$$
\times \mathcal{V}\left(t, \mathbf{x}_{k+1}\right) g\left(\mathbf{x}_{k+1}, t_{k+1} \mid \mathbf{x}_{k}, t_{k}\right) d \mathbf{x}_{k+1},
$$

where $\mathcal{V}\left(t ; \mathbf{x}_{k+1}, t_{k+1}\right)=\int V_{j}(\mathbf{x}, t) g\left(\mathbf{x}, t \mid \mathbf{x}_{k+1}, t_{k+1}\right) d \mathbf{x}$.

Because the velocity defined by Eq. (5) is always finite, the function $\mathcal{V}\left(t ; \mathbf{x}_{k+1}, t_{k+1}\right)$ is bounded, i.e., there exists an $M>0$ so that $\mathcal{V}\left(t ; \mathbf{x}_{k+1}, t_{k+1}\right) \leq M$ for all $\mathbf{x}_{k+1} \in \mathbb{R}^{3}$ and $t_{k+1}$ $\in \mathbb{R}$. By using Eq. (8) for $i=j$ and the Cauchy-Schwarz inequality in the form

$$
\begin{aligned}
& \left(\int_{\left|\mathbf{x}^{\prime}-\mathbf{x}\right| \geq \epsilon}\left|x_{i}\right| g\left(\mathbf{x}^{\prime}, t+\Delta t \mid \mathbf{x}, t\right) d \mathbf{x}^{\prime}\right)^{2} \\
& \leq \int_{\left|\mathbf{x}^{\prime}-\mathbf{x}\right| \geq \epsilon} x_{i}^{2} g\left(\mathbf{x}^{\prime}, t+\Delta t \mid \mathbf{x}, t\right) d \mathbf{x}^{\prime} \\
& \quad \times \int_{\left|\mathbf{x}^{\prime}-\mathbf{x}\right| \geq \epsilon} g\left(\mathbf{x}^{\prime}, t+\Delta t \mid \mathbf{x}, t\right) d \mathbf{x}^{\prime}
\end{aligned}
$$

one obtains the condition

$$
\lim _{\Delta t \rightarrow 0} \frac{1}{\Delta t} \int_{\left|\mathbf{x}^{\prime}-\mathbf{x}\right| \geq \epsilon}\left|x_{i}^{\prime}\right| g\left(\mathbf{x}^{\prime}, t+\Delta t \mid \mathbf{x}, t\right) d \mathbf{x}^{\prime}=0 .
$$

Computing the last integral in Eq. (A4) as sum between the integral over the sphere of radius $\epsilon$ and the integral outside the sphere, we have

$$
\begin{aligned}
& \mid \int_{\left|\mathbf{x}_{k+1}-\mathbf{x}_{k}\right| \geq \epsilon}\left(x_{k+1, i}-x_{k, i}\right) \\
& \quad \times \mathcal{V}\left(t ; \mathbf{x}_{k+1}, t_{k+1}\right) g\left(\mathbf{x}_{k+1}, t_{k+1} \mid \mathbf{x}_{k}, t_{k}\right) d \mathbf{x}_{k+1} \mid \\
& \leq M \int_{\left|\mathbf{x}_{k+1}-\mathbf{x}_{k}\right| \geq \epsilon}\left|\left(x_{k+1, i}-x_{k, i}\right)\right| \\
& \quad \times g\left(\mathbf{x}_{k+1}, t_{k}+\Delta t \mid \mathbf{x}_{k}, t_{k}\right) d \mathbf{x}_{k+1}^{\rightarrow} 0 \\
& \quad 0
\end{aligned}
$$

due to condition (A5). Considering the negative and positive parts and applying the theorem of mean, the integral over the sphere of radius $\epsilon$ can be computed as

$$
\begin{gathered}
\int_{\left|\mathbf{x}_{k+1}-\mathbf{x}_{k}\right|<\epsilon}\left(x_{k+1, i}-x_{k, i}\right) \mathcal{V}\left(t ; \mathbf{x}_{k+1}, t_{k+1}\right) g\left(\mathbf{x}_{k+1}, t_{k+1} \mid \mathbf{x}_{k}, t_{k}\right) d \mathbf{x}_{k+1} \\
=\mathcal{V}\left(t ; \mathbf{x}_{k}, t_{k+1}\right) \int_{\left|\mathbf{x}_{k+1}-\mathbf{x}_{k}\right|<\epsilon}\left(x_{k+1, i}-x_{k, i}\right) \\
\quad \times g\left(\mathbf{x}_{k+1}, t_{k}+\Delta t \mid \mathbf{x}_{k}, t_{k}\right) d \mathbf{x}_{k+1}+\mathcal{O}\left(\epsilon^{2}\right)
\end{gathered}
$$

For $\Delta t \rightarrow 0$, from Eq. (5) and Eqs. (A4)-(A7) one obtains

$$
\begin{aligned}
& \int c\left(\mathbf{x}_{0}, t_{0}\right) d \mathbf{x}_{0} \int\left(x_{i}-x_{0 i}\right) V_{j}(\mathbf{x}, t) g\left(\mathbf{x}, t \mid \mathbf{x}_{0}, t_{0}\right) d \mathbf{x} \\
&= \int c\left(\mathbf{x}_{0}, t_{0}\right) d \mathbf{x}_{0} \int_{0}^{t} d t^{\prime} \iint V_{j}(\mathbf{x}, t) V_{i}\left(\mathbf{x}^{\prime}, t^{\prime}\right) \\
& \quad \times g\left(\mathbf{x}, t \mid \mathbf{x}^{\prime}, t^{\prime}\right) g\left(\mathbf{x}^{\prime}, t^{\prime} \mid \mathbf{x}_{0}, t_{0}\right) d \mathbf{x} d \mathbf{x}^{\prime}+\mathcal{O}\left(\boldsymbol{\epsilon}^{2}\right) .
\end{aligned}
$$

Finally, passing to the limit $\epsilon \rightarrow 0$, we have

$$
\begin{gathered}
\int c\left(\mathbf{x}_{0}, t_{0}\right) d \mathbf{x}_{0} \int\left(x_{i}-x_{0 i}\right) V_{j}(\mathbf{x}, t) g\left(\mathbf{x}, t \mid \mathbf{x}_{0}, t_{0}\right) d \mathbf{x} \\
=\int c\left(\mathbf{x}_{0}, t_{0}\right) d \mathbf{x}_{0} \int_{0}^{t} d t^{\prime} \iint V_{j}(\mathbf{x}, t) V_{i}\left(\mathbf{x}^{\prime}, t^{\prime}\right) \\
\times g\left(\mathbf{x}, t \mid \mathbf{x}^{\prime}, t^{\prime}\right) g\left(\mathbf{x}^{\prime}, t^{\prime} \mid \mathbf{x}_{0}, t_{0}\right) d \mathbf{x} d \mathbf{x}^{\prime} .
\end{gathered}
$$

Replacing in the second term of Eq. (A3) the contribution [Eq. (A8)] and the similar one obtained by permutation of the indices $i$ and $j$ one obtains Eq. (11) in the main text.

\section{APPENDIX B: LAGRANGIAN DESCRIPTION}

The expectation $\langle f(\mathbf{X}(t))\rangle=\int f(\mathbf{x}) c(\mathbf{x}, t) d \mathbf{x}$ of some function with compact support $f(\mathbf{x})$ can be written by using the Dirac $\delta$ distribution as

$$
\left\langle\int f(\mathbf{x}) \delta[\mathbf{x}-\mathbf{X}(t)] d \mathbf{x}\right\rangle=\int f(\mathbf{x})\langle\delta[\mathbf{x}-\mathbf{X}(t)]\rangle d \mathbf{x} .
$$

Hence, the one-point probability density (i.e., the normalized concentration) can formally be written as $c(\mathbf{x}, t)=\langle\delta[\mathbf{x}$ $-\mathbf{X}(t)]\rangle$, and the $n$-point joint densities as expectations of products of delta functions (e.g., [39]).

Similarly, the transition probability density is the conditional expectation $g\left(\mathbf{x}, t \mid \mathbf{x}_{0}, t_{0}\right)=\left\langle\delta[\mathbf{x}-\mathbf{X}(t)] \mid \mathbf{X}\left(t_{0}\right)\right\rangle=\langle\delta[\mathbf{x}$ $-\mathbf{X}(t)]\rangle_{D}$, where by angular brackets with subscript $D$ we denoted the conditional expectation for fixed initial positions $\mathbf{X}(0)$ of the particles. For the purpose of a transparent Lagrangian description it is also convenient to use the subscript $X_{0}$ for averages with respect to initial positions. With these, the concentration [Eq. (3)] can be expressed as

$$
c(\mathbf{x}, t)=\int g\left(\mathbf{x}, t \mid \mathbf{x}_{0}, t_{0}\right) c\left(\mathbf{x}_{0}, t_{0}\right) d \mathbf{x}_{0}=\langle\delta[\mathbf{x}-\mathbf{X}(t)]\rangle_{D X_{0}} .
$$

In the same way, for higher order probability densities one obtains

$$
\begin{aligned}
& p\left(\mathbf{x}_{n}, t_{n} ; \mathbf{x}_{n-1}, t_{n-1} ; \ldots ; \mathbf{x}_{1}, t_{1}\right) \\
& \quad=\int p\left(\mathbf{x}_{n}, t_{n} ; \mathbf{x}_{n-1}, t_{n-1} ; \ldots ; \mathbf{x}_{1}, t_{1} \mid \mathbf{x}_{0}, 0\right) c\left(\mathbf{x}_{0}, t_{0}\right) d \mathbf{x}_{0} \\
& \quad=\left\langle\delta\left[\mathbf{x}_{n}-\mathbf{X}\left(t_{n}\right)\right] \delta\left[\mathbf{x}_{n-1}-\mathbf{X}\left(t_{n-1}\right)\right] \ldots \delta\left[\mathbf{x}_{n}-\mathbf{X}\left(t_{n}\right)\right]\right\rangle_{D X_{0}} .
\end{aligned}
$$


[1] C. W. Gardiner, Stochastic Methods: A Handbook for the Natural and Social Sciences (Springer, Berlin, 2009).

[2] R. Zwanzig, Phys. Rev. 124, 983 (1961).

[3] A. V. Mokshin, R. M. Yulmetyev, and P. Hänggi, Phys. Rev. Lett. 95, 200601 (2005).

[4] R. Balescu, Plasma Phys. Controlled Fusion 42, B1 (2000).

[5] R. Morgado, F. A. Oliveira, G. G. Batrouni, and A. Hansen, Phys. Rev. Lett. 89, 100601 (2002).

[6] F. Piazza and S. Lepri, Phys. Rev. B 79, 094306 (2009).

[7] M. A. Despósito and A. D. Viñales, Phys. Rev. E 77, 031123 (2008).

[8] F. N. C. Paraan, M. P. Solon, and J. P. Esguerra, Phys. Rev. E 77, 022101 (2008).

[9] A. J. Majda and P. R. Kramer, Phys. Rep. 314, 237 (1999).

[10] C. Aslangul, J.-Ph. Bouchaud, A. Georges, N. Pottier, and D. Saint-James, J. Stat. Phys. 55, 461 (1989).

[11] J. Eberhard, N. Suciu, and C. Vamoş, J. Phys. A: Math. Theor. 40, 597 (2007).

[12] J. L. Doob, Stochastic Processes (Wiley, New York, 1990).

[13] P. E. Kloeden and E. Platen, Numerical Solutions of Stochastic Differential Equations (Springer, Berlin, 1999).

[14] J.-P. Bouchaud, A. Georges, J. Koplik, A. Provata, and S. Redner, Phys. Rev. Lett. 64, 2503 (1990).

[15] J.-P. Bouchaud and A. Georges, Phys. Rep. 195, 127 (1990).

[16] P. Castiglione, J. Phys. A 33, 1975 (2000).

[17] C. L. Zirbel, Adv. Appl. Probab. 33, 810 (2001).

[18] N. Suciu, C. Vamoş, H. Vereecken, K. Sabelfeld, and P. Knabner, Revue d'Analyse Numérique et de Théorie de l'Approximation 37, 221 (2008).

[19] A. Fannjiang and T. Komorowski, Ann. Appl. Probab. 9, 591 (1999).

[20] P. K. Kitanidis, J. Hydrol. 102, 453 (1988).

[21] N. Suciu, C. Vamoş, H. Vereecken, K. Sabelfeld, and P. Knabner, Mathematics Department, Friedrich-Alexander University Erlangen-Nuremberg, Preprint No. 324, 2008, url: http:// www.am.uni-erlangen.de/en/prepreprints.2000.html
[22] H. Kesten and G. C. Papanicolaou, Commun. Math. Phys. 65, 97 (1979).

[23] N. Suciu, C. Vamoș, and J. Eberhard, Water Resour. Res. 42, W11504 (2006).

[24] N. Suciu (unpublished).

[25] N. Suciu, C. Vamoş, H. Vereecken, K. Sabelfeld, and P. Knabner, Water Resour. Res. 44, W08501 (2008).

[26] G. Sposito and G. Dagan, Water Resour. Res. 30, 585 (1994).

[27] C. Vamoş, Ş. Şoltuz, and M. Crăciun, e-print arXiv:0709.2963.

[28] N. Suciu and C. Vamoş, in Monte Carlo and Quasi-Monte Carlo Methods 2008, edited by P. L'Ecuyer and A. B. Owen (Springer, Heidelberg, to be published).

[29] C. L. Winter, C. M. Newman, and S. P. Neuman, SIAM J. Appl. Math. 44, 411 (1984).

[30] M. G. Trefry, F. P. Ruan, and D. McLaughlin, Water Resour. Res. 39, 1063 (2003)

[31] A. Compte and M. O. Cáceres, Phys. Rev. Lett. 81, 3140 (1998).

[32] C. Vamoş, N. Suciu, and H. Vereecken, J. Comput. Phys. 186, 527 (2003)

[33] N. Suciu, C. Vamoş, J. Vanderborght, H. Hardelauf, and H. Vereecken, Water Resour. Res. 42, W04409 (2006).

[34] P. Le Doussal and J. Machta, Phys. Rev. B 40, 9427 (1989).

[35] N. Suciu and C. Vamoş, Water Resour. Res. 43, W12601 (2007).

[36] A. M. Yaglom, Correlation Theory of Stationary and Related Random Functions, Basic Results Vol. I (Springer, New York, 1987).

[37] C. Vamoş, N. Suciu, H. Vereecken, J. Vanderborht, and O. Nitzsche, Forschungszentrum Jülich Report No. ICGIV.00501, 2001 (unpublished).

[38] S. C. Ying, I. Vattulainen, J. Merikoski, T. Hjelt, and T. AlaNissila, Phys. Rev. B 58, 2170 (1998).

[39] N. G. van Kampen, Stochastic Processes in Physics and Chemistry (North-Holland, Amsterdam, 1981). 